Original Paper http://ajol.info/index.php/ijbcs http://indexmedicus.afro.who.int

\title{
Distribution des ligneux sur le tracé de la grande muraille verte : cas de batha et de wadi-fira Ouest au Tchad
}

\author{
Mahamat ABAKAR GUIHINI', Mariama Dalanda DIALLO ${ }^{2,3,4}$, Aly DIALLO', \\ Minda MAHAMAT SALEH ${ }^{1}$ et Aliou GUISSE ${ }^{1,3,4}$
}

\author{
'Département de Biologie Végétale, Faculté des Sciences et Techniques, Université Cheikh Anta Diop de \\ Dakar, B.P 5005 Dakar, Sénégal. \\ ${ }^{2}$ Section Productions Végétales et Agronomies, UFR des Sciences Agronomiques, de l'Aquaculture et des \\ Technologies Alimentaires, Université Gaston Berger de Saint Louis, B.P 234 Saint Louis, Sénégal. \\ ${ }^{3}$ Observatoire Homme Milieu Tessékéré, Sénégal, UCAD/CNRS, Dakar, Sénégal. \\ ${ }^{4}$ Unité Mixte Internationale 3189 « Environnement, Santé, Société », Dakar, Sénégal. \\ *Auteur correspondant ; E-mail : abakar.guihini@yahoo.fr ; Tél : +(235) 66502929
}

\begin{tabular}{lll}
\hline Received: 18- 11-2020 & Accepted: 28- 01-2021 & Published: 28- 02- 2021 \\
\hline
\end{tabular}

\section{RESUME}

Les déficits pluviométriques combinés à l'action anthropique ont entrainé une dégradation des ressources naturelles à partir desquelles les paysans tchadiens tirent leurs moyens de subsistance. Cette étude avait pour objectif de déterminer la composition floristique, la circonférence et la hauteur des peuplements ligneux dans deux sites de la Grande Muraille Verte du Tchad (Batha et Wadi-Fira Ouest). Pour cela, nous avons utilisé la méthode des relevés floristiques et des mesures dendrométriques pour caractériser la végétation. Cette méthode nous a permis d'inventorier 15 espèces réparties en 11 genres et 6 familles. Les espèces les plus dominantes sont Balanites aegyptiaca (Delile), Acacia tortilis (Forssk.) Hayne subsp. raddiana (Savi) Brenan et Capparis decidua Edgew (Forssk.). La répartition des individus selon la grosseur et la hauteur permettent d'établir la structure du peuplement ligneux. Ainsi, dans le Batha, le peuplement ligneux ainsi que les espèces dominantes (Balanites aegyptiaca, Acacia raddiana et Capparis decidua) est constitué de cinquante-deux virgule seize pour cent $(52,16 \%)$ d'individus qui ont une circonférence comprise entre 10 et 50 $\mathrm{cm}$. Au niveau de Wadi-Fira Ouest, le peuplement est dominé par des individus de circonférence comprise entre 20 et $60 \mathrm{~cm}$. Le peuplement du Batha renferme des individus qui ont une hauteur comprise entre 1 et 14 m. Quatre-vingt-six virgule soixante-sept pour cent $(86,67 \%)$ des espèces ont une hauteur inférieure ou égale à $7 \mathrm{~m}$. Tandis que dans le Wadi-Fira Ouest, la hauteur des individus du peuplement varie de 0,9 à $12 \mathrm{~m}$ et quatrevingt-dix pour cent $(90 \%)$ des individus ont une hauteur comprise entre 0,9 et $7 \mathrm{~m}$. La strate ligneuse est dominée par des arbustes en grande partie. L'étude a permis de mettre en évidence que les facteurs d'ordre climatique et anthropique ont un impact sur l'environnement des sites.

(C) 2021 International Formulae Group. All rights reserved.

Mots clés : Ligneux, Circonférence, Hauteur, Batha, Wadi-Fira Ouest, Tchad 


\title{
Distribution of woody trees along the route of the great green wall: cases of batha and wadi-fira West in Chad
}

\begin{abstract}
Rainfall deficits combined with anthropogenic action have led to the degradation of the natural resources from which chadian peasants derive their livelihoods. The objective of this study was to determine the floristic composition, circumference and height of woody stands in two sites of Chad's Great Green Wall (Batha and Wadi-Fira West). For this purpose, we used the method of floristic surveys and dendrometric measurements to characterize the vegetation. This method allowed us to inventory 15 species divided into 11 genera and 6 families. The most dominant species are Balanites aegyptiaca (Delile), Acacia tortilis (Forssk.) Hayne subsp. raddiana (Savi) Brenan and Capparis decidua Edgew (Forssk.). The distribution of individuals according to size and height makes it possible to establish the structure of the woody stand. Thus, in the Batha, the woody stand as well as the dominant species (Balanites aegyptiaca, Acacia raddiana and Capparis decidua) consists of fifty-two point sixteen percent (52.16\%) of individuals that have a circumference between 10 and $50 \mathrm{~cm}$. At Wadi-Fira West, the stand is dominated by individuals with circumference between 20 and $60 \mathrm{~cm}$. The Batha stand contains individuals that are between 1 and $14 \mathrm{~m}$ high. Eighty-six point sixty-seven percent $(86.67 \%$ ) of the species are less than or equal to $7 \mathrm{~m}$ in height. While in Wadi-Fira West, the height of individuals in the stand varies from 0.9 to $12 \mathrm{~m}$ and ninety percent $(90 \%)$ of the individuals are between 0.9 and $7 \mathrm{~m}$ in height. The woody stratum is dominated by shrubs for the most part. The study showed that climatic and anthropogenic factors have an impact on the environment of the sites.
\end{abstract}

(C) 2021 International Formulae Group. All rights reserved.

Keywords: Woody, Circumference, Height, Batha, Wadi-Fira West, Chad.

\section{INTRODUCTION}

Le Tchad, à l'instar des autres pays sahélo-sahariens, a connu ces dernières années une explosion démographique (6 288261 en 1993 contre 12,3 millions en 2013) (INSEED, 2013) qui a engendré une influence considérable sur les ressources naturelles. En effet, la zone sahélienne subit depuis quelques années des variabilités climatiques qui, combinés à des actions humaines, ont entrainé une forte dégradation des ressources naturelles (Ndong et al., 2015) et une baisse des productions agrosylvopastorales (SNPA/GMV, 2012). Malgré l'importance reconnue des végétaux qui rendent beaucoup de services à la population humaine (Kaou et al., 2017), ils constituent des sources alimentaires essentielles pour les animaux (Sarr et al., 2013). Ils créent un microclimat qui favorise les cultures et protège les sols contre les érosions (Ngom et al., 2018). Cependant, la disparition de la biodiversité se poursuit et constitue une menace pour l'humanité (Sinsin et Kampmann, 2010). Tous ces facteurs ont fait que beaucoup d'efforts ont été fournis pour la protection et la restauration de ces écosystèmes fragiles notamment avec la mise en place de la Grande Muraille Verte dont l'objectif principal est de permettre de faire face à la désertification et de trouver des solutions alternatives aux changements climatiques (Goffner et JeanLuc, 2020).

Dans l'optique de caractériser la Grande Muraille Verte, quelques études ont été menées sur les caractéristiques des sols, de la flore et de la végétation ligneuse et herbacé (Diallo et al., 2011; Ndiaye et al., 2013; Mahamat Saleh et al., 2015b ; Diallo et al., 2019; Talla et al., 2020) et sur la caractérisation de la végétation ligneuse et son usage par les populations locales (Niang et al., 2014) dans la zone sahélienne du Sénégal et du Tchad. Cependant, il faut noter que peu d'investigations ont été faites sur la partie tchadienne de la Grande Muraille Verte en ce qui concerne la caractérisation de la végétation et des sols (Mahamat Saleh et al., 2015a et $b, 2016)$. L'objectif global de cette 
étude était de caractériser la végétation ligneuse dans la partie ouest du tracé de la GMV du Tchad (Batha et de Wadi-Fira). Il s'agit spécifiquement de déterminer l'importance de la diversité floristique et la structure de la végétation ligneuse.

\section{MATÉRIEL ET MÉTHODES Site de l'étude}

La présente étude a été faite dans les sites de Batha et de Wadi-Fira Ouest du tracé de la Grande Muraille Verte au Tchad (Figure 1). La zone d'étude fait une longueur de 277 $\mathrm{km}$ sur $15 \mathrm{~km}$ de largeur, soit une superficie totale de 415500 ha.

Le climat est de type sahélien aride à deux saisons : une saison sèche qui s'étend d'octobre à mai et une saison pluvieuse de juin à septembre (Bechir et Mopate, 2015). Cette zone reçoit en moyenne 202,6 mm de pluie, avec des variations considérables d'une année à l'autre (ANAM, 2020). La température moyenne annuelle est de $29{ }^{\circ} \mathrm{C}$ avec un maximum absolu de $41,7^{\circ} \mathrm{C}$ (mai) et un minimum absolu de $14,5{ }^{\circ} \mathrm{C}$ (janvier) (ANAM, 2020).

Le choix des sites est motivé par les différentes unités pédologiques et de végétations qui y sont rencontrées. En effet, les sites sont caractérisés par des sols à texture sableuse, sablo-argileuse et argilo-limonosableuse selon les unités pédologiques SNPA/GMV (2012). En outre, les études dans ces sites permettront de compléter les travaux qui ont été déjà réalisé dans la partie précédente du tracé (Lac, Kanem et Bahr El Gazel). La végétation est constituée par une steppe arbustive à épines et une strate herbacée qui est dominée par des graminées annuelles (Bernabé, 2013).

\section{Méthodologie}

Pour caractériser la végétation ligneuse de cette zone, nous avons utilisé la méthode des relevés floristiques et des mesures dendrométriques. En juin 2018, nous avons procédé à un inventaire floristique et des mesures des données écologiques sur les individus rencontrés dans des placettes ou unités d'échantillonnage des différents sites. Les placettes d'échantillonnage ont été installés de manière aléatoire comme suggéré par Niang et al. (2014).

L'échantillonnage a consisté à répartir de façon aléatoire des placettes de $50 \mathrm{~m} \mathrm{X} 50$ $\mathrm{m}$, géo-référencées en utilisant la méthode 3 $4-5$ pour retrouver les angles droits. Cette surface correspondra à l'aire minimale pour l'étude de la végétation ligneuse sahélienne (Boudet, 1984).

Nous avons effectué des relevés floristiques à partir d'un échantillon de 59 placettes de $2500 \mathrm{~m}^{2}$ de surface chacune (Ndiaye et al., 2013; Mahamat Saleh et al., 2015b ; Abakar Guihini et al., 2018). Ces placettes sont réparties au hasard dans l'unité de végétation et dans chaque site en fonction des unités morpho pédologiques (dune, plateau et dépression) : 42 placettes à Batha et 17 à Wadi-Fira Ouest. Cette différence sur la répartition des placettes dans les deux sites s'explique par l'homogénéité de la végétation de Wadi-fira Ouest et son hétérogénéité à Batha. Tous les individus rencontrés à l'intérieur de ces placettes ont été recensés et identifiés.

La détermination des taxons a été effectuée à l'aide de la Flore du Sénégal (Berhaut, 1979) et des Arbres et Arbustes du Sahel (Maydell, 1983). Les synonymes ont été actualisés d'après l'énumération des plantes à fleurs d'Afrique tropicale (Lebrun et Stork, 1997).

Pour chaque individu rencontré, plusieurs paramètres ont été mesurés dont :

* les circonférences ou diamètres des arbres à $0,30 \mathrm{~m}$ du sol et à 1,30 m du sol ;

* la hauteur (H) des arbres depuis le sol jusqu'à la cime et celle de la $1^{\text {ere }}$ branche vivante.

\section{Traitement des données}

Les données obtenues ont été saisies et traitées avec le tableur Excel. Les formules cidessous nous ont permis de calculer ces paramètres : 


\section{Composition \\ Les données collectées ont été} analysées et la liste des espèces inventoriées ainsi que leurs taxonomies dressées afin d'évaluer la composition floristique.

La fréquence spécifique, nous renseigne sur la distribution d'une espèce dans un peuplement et est exprimée en pourcentage. Elle est estimée par la formule suivante :

\section{$\mathrm{F}=\mathrm{Ni} / \mathrm{Nr} X 100$}

F : fréquence spécifique exprimée en pourcentage ;

$\mathrm{Ni}$ : nombre de relevés où l'on retrouve l'espèce $\mathrm{i}$; et
$\mathrm{Nr}$ : nombre total de relevés.

\section{$>\quad$ Distribution selon la hauteur et la grosseur}

Avec la classe de circonférence et de hauteur, les densités relatives ont été calculées dans le but de déterminer les distributions des arbres selon la grosseur et la hauteur. La répartition des individus selon la grosseur a permis d'évaluer la structure horizontale. Quant à la structure verticale, elle est appréciée par leur distribution selon la hauteur. Le nom "arbustes" a été donné aux individus adultes dont la hauteur ne dépasse pas $7 \mathrm{~m}$ et "arbres" à ceux dont la hauteur dépasse cette valeur (Touffet, 1982).

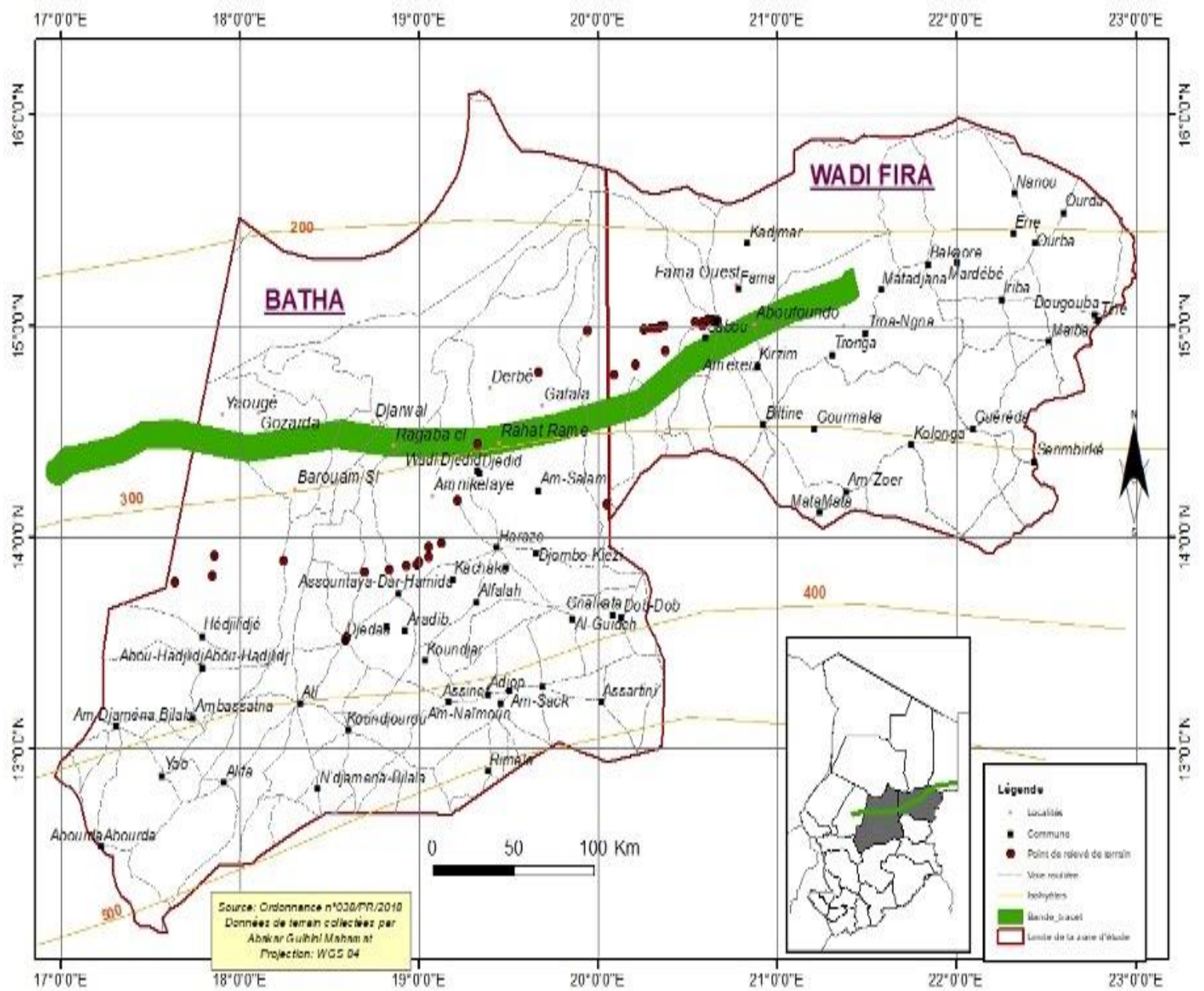

Figure 1 : Localisation de la zone d'étude. 


\section{RÉSULTATS \\ Composition et diversité floristique}

La flore ligneuse inventoriée de la zone d'étude est riche de 15 espèces, réparties entre 11 genres, appartenant à 6 familles (Tableau 1). La famille de Mimosaceae (6 espèces) est la plus diversifiée, suivie de celle des Capparaceae (3 espèces). Les Apocynaceae et les Rhamnaceae sont représentées par 2 espèces chacune. Toutes les autres familles ne comptent qu'une seule espèce, il s'agit des Balanitaceae et des Salvadoraceae.

Le genre Acacia est mieux représenté, suivi de Vachellia et Ziziphus qui sont représentés par deux (2) espèces. Tous les autres genres renferment chacun une (1) espèce (Tableau 1). Le site de Batha avec 14 espèces, 11 genres et 6 familles est plus riche que le site de Wadi-Fira Ouest qui dispose de 10 espèces, 7 genres et 5 familles. Les espèces Acacia ehrenbergiana, Acacia senegal, Leptadenia pyrotechnica, Maerrua crassifolia et Salvadora persica ne sont rencontrées qu'à Batha alors que Acacia laeta est uniquement retrouvé à Wadi-Fira Ouest. (Tableau 1).

\section{Fréquence des espèces}

Les espèces les plus fréquentes dans les zones d'étude sont Balanites aegyptiaca (86,98\%), Acacia raddiana (64,57\%), Capparis decidua (56,87\%), Acacia senegal (45,24\%), Ziziphus mauritania (35,5\%), Acacia nilotica (32,50\%), Boscia senegalensis (30,16\%) et Acacia seyal $(24,23 \%)$.

Plus spécifiquement, au niveau du site de Batha, Balanites aegyptiaca (97,62\%), Acacia raddiana (92,86\%), Capparis decidua (88,10\%), Acacia senegal (78,57\%), Boscia senegalensis $(73,81 \%)$, Ziziphus mauritania $(66,67 \%)$, Acacia nilotica $(61,90 \%)$ et Acacia seyal $(59,52 \%)$ sont les espèces les plus fréquentes. Dans le Wadi-Fira Ouest, les espèces les plus fréquentes sont Balanites aegyptiaca $(94,12 \%), \quad$ Acacia raddiana (82,35\%), Capparis decidua (76,47\%), Acacia nilotica et Boscia senegalensis (70,59\%), Ziziphus mauritania (64,71\%), Acacia seyal et Ziziphus spina $(58,82 \%)$.

\section{Distribution des individus selon la circonférence}

L'étude a permis de répertorier 457 individus dans la zone d'étude, soit une densité réelle de 30,95 individus à l'hectare. Ces individus sont répartis de la manière suivante dans les sites de l'étude : 346 individus pour le site du Batha et 111 pour le site de Wadi-Fira Ouest.

La répartition des individus selon la grosseur, montre la structure démographique du peuplement ligneux. Dans le Batha, le peuplement ligneux ainsi que les espèces dominantes de la zone (Balanites aegyptiaca, Acacia raddiana et Capparis decidua) sont constitués de plus de $50 \%$ d'individus ayant moins de $50 \mathrm{~cm}$ de circonférence (Figure 2 A). Le peuplement de ce site est constitué de quatre-vingt-sept virgule cinquante-neuf pour cent $(87,59 \%)$ des individus qui ont une circonférence comprise entre 10 et $100 \mathrm{~cm}$. La circonférence des individus du peuplement dans ce site varie de 10 à $200 \mathrm{~cm}$. La population d'Acacia raddiana, de Balanites aegyptiaca et de Capparis decidua sont respectivement comprises entre les circonférences de 10 à $140 \mathrm{~cm}, 10$ à $110 \mathrm{~cm}$ et de 10 à $100 \mathrm{~cm}$. Aucune des espèces dominantes de la zone d'étude n'a dépassé les $140 \mathrm{~cm}$ de circonférence.

Cependant, dans le site de Wadi-Fira Ouest, les espèces dominantes ainsi que le peuplement présentent majoritairement des arbres de circonférence comprise entre 20 et $60 \mathrm{~cm}$ (Figure $2 \mathrm{~B}$ ). La circonférence des individus à Wadi-Fira Ouest varie de 10 à 210 $\mathrm{cm}$. Ce peuplement est constitué de $82,89 \%$ des individus ayant une circonférence comprise entre 10 et $100 \mathrm{~cm}$. Quant à la population d'Acacia raddiana, elle est constituée d'individus dont la circonférence est comprise entre 10 et $110 \mathrm{~cm}$. En ce qui concerne Balanites aegyptiaca, le diamètre varie de 10 à $90 \mathrm{~cm}$ et pour Capparis decidua, il va de 10 à $80 \mathrm{~cm}$.

A Batha, la classe de moins de $50 \mathrm{~cm}$ renferme $52,15 \%$ (Figure $3 \mathrm{~A}$ ) des individus. L'essentiel de ces individus se trouvent dans la classe $[20,30 \mathrm{~cm}],[10,20 \mathrm{~cm}],[40,30 \mathrm{~cm}]$ 
et $[30,40 \mathrm{~cm}]$ qui représentent respectivement $14,69 \% ; 12,97 \% ; 12,39 \%$ et $12,1 \%$ de la population totale des ligneux répertoriée de la zone d'étude.

$\mathrm{Au}$ niveau de Wadi-Fira Ouest, la classe des individus de moins de $50 \mathrm{~cm}$ renferme 46,85\% (Figure 3 B) des individus. L'essentiel de ces individus sont contenus dans les classes de $[10,20 \mathrm{~cm}],[20,30 \mathrm{~cm}]$, $[30,40 \mathrm{~cm}],[40,50 \mathrm{~cm}]$ et $[60,70 \mathrm{~cm}]$ avec respectivement des pourcentages de $7,21 \%$; $15,32 \% ; 12,61 \% ; 11,71 \%$ et $10,81 \%$ de la population totale des ligneux répertoriés de la zone étudiée.

\section{Distribution des individus selon la hauteur}

Le peuplement du Batha est constitué de plus de $70 \%$ d'individus ayant une hauteur comprise entre 2 et $7 \mathrm{~m}$. Les espèces dominantes de ce site présentent des individus qui n'atteignent pas $8 \mathrm{~m}$ de hauteur (Figure 4 A). Dans le Wadi-Fira Ouest, le peuplement est constitué de plus de $60 \%$ d'individus ayant entre 3 et $6 \mathrm{~m}$ de hauteur (Figure $4 \mathrm{~B}$ ). Les espèces dominantes du site ont toutes une hauteur inférieure à $6 \mathrm{~m}$. Les individus dont la hauteur supérieure à $8 \mathrm{~m}$ sont représentés avec
$7,81 \%$ et $6,3 \%$ de peuplement respectivement à Batha et Wadi-Fira Ouest.

La hauteur des individus varie de 1 à $14 \mathrm{~m}$ dans le site de Batha. Quatre-vingt-six virgule soixante-sept pour cent $(86,67 \%)$ des espèces ont une hauteur inférieure à $7 \mathrm{~m}$ (Figure 5). Les hauteurs de la population d'Acacia raddiana et Balanites aegyptiaca sont comprises entre 1 et $8 \mathrm{~m}$ contrairement à Capparis decidua qui présente des individus dont la hauteur ne dépasse pas $7 \mathrm{~m}$.

Dans le Wadi-Fira Ouest, la hauteur des individus du peuplement varie de 0,9 à 12 m. Quatre-vingt-dix pour cent $(90 \%)$ des individus ont une hauteur comprise entre 0,9 et $7 \mathrm{~m}$ (Figure 6). La classe de [4,5 m] est la mieux représentée avec $26,1 \%$, suivie de celle de $[3,4 \mathrm{~m}]$ et $[5,6 \mathrm{~m}]$ qui renferme chacune $20,7 \%$ des individus répertoriés du site étudié (Figure 6). Quant aux espèces dominantes (Balanites aegyptiaca, Acacia raddiana et Capparis decidua), elles présentent toutes des individus qui ont une hauteur comprise entre 1 et $6 \mathrm{~m}$.

Tableau 1 : Liste des espèces ligneuses recensées dans la zone d'étude.

\begin{tabular}{|c|c|c|c|}
\hline \multirow[b]{2}{*}{ Familles } & \multirow[b]{2}{*}{ Espèces } & \multicolumn{2}{|c|}{ Sites } \\
\hline & & Batha & $\begin{array}{c}\text { Wadi-Fira } \\
\text { Ouest }\end{array}$ \\
\hline \multirow{6}{*}{ Mimosaceae } & Acacia nilotica (L.) Willd. ex Delile & $*$ & $*$ \\
\hline & Acacia Senegal (L.) Willd. & $*$ & \\
\hline & Acacia seyal auct. & $*$ & $*$ \\
\hline & Acacia raddiana Savi. & $*$ & $*$ \\
\hline & Acacia laeta $\mathrm{R}$. Br. Ex Benth. & & $*$ \\
\hline & Acacia ehrenbergiana Rein chenb. Ex Benth. & $*$ & \\
\hline \multirow{3}{*}{ Capparaceae } & Boscia senegalensis (Pers.) Lam. ex Poir. & $*$ & $*$ \\
\hline & Capparus decidua (Forssk.) Edgew. & $*$ & $*$ \\
\hline & Maerrua crassifolia Forssk & $*$ & \\
\hline Apocynaceae & Calotropis procera (Aiton) W.T. Aiton & * & * \\
\hline
\end{tabular}




\begin{tabular}{llll}
\cline { 2 - 4 } Reptadenia pyrotechnica (Forssk.) Decne. & $*$ & \\
\hline \multirow{2}{*}{ Rhamnaceae } & Ziziphus mauritania auct. & $*$ & $*$ \\
\cline { 2 - 4 } & Ziziphus spina Christi (L) Desf. & $*$ & $*$ \\
\hline Balanitaceae & Balanites aegyptiaca (L.) Delile & $*$ & $*$ \\
\hline Salvadoraceae & Salvadora persica L. & $*$ & \\
\hline
\end{tabular}

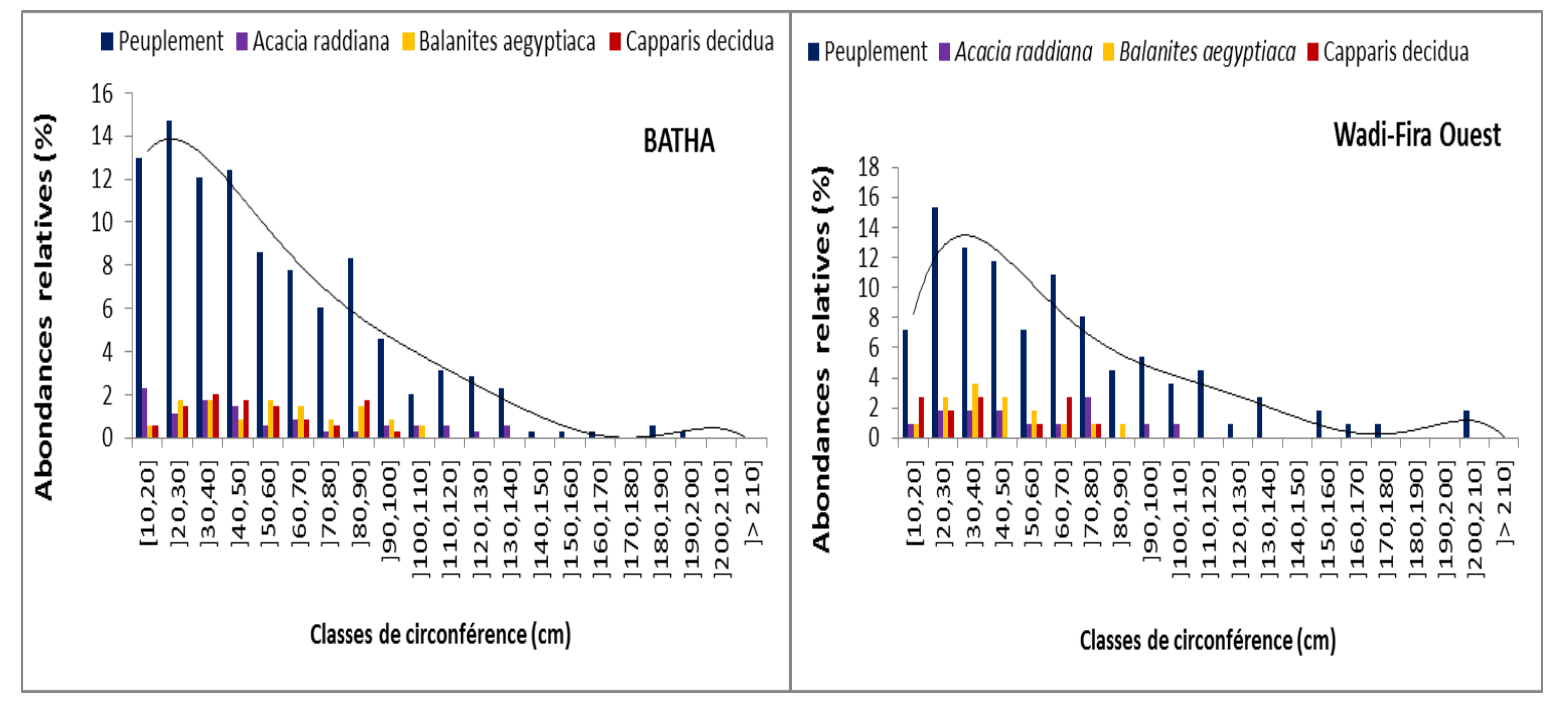

Figure 2 : Répartition des individus du peuplement ligneux et des espèces dominantes selon la circonférence à $0,30 \mathrm{~m}(\mathrm{~A})$ à Batha et (B) à Wadi-Fira Ouest.

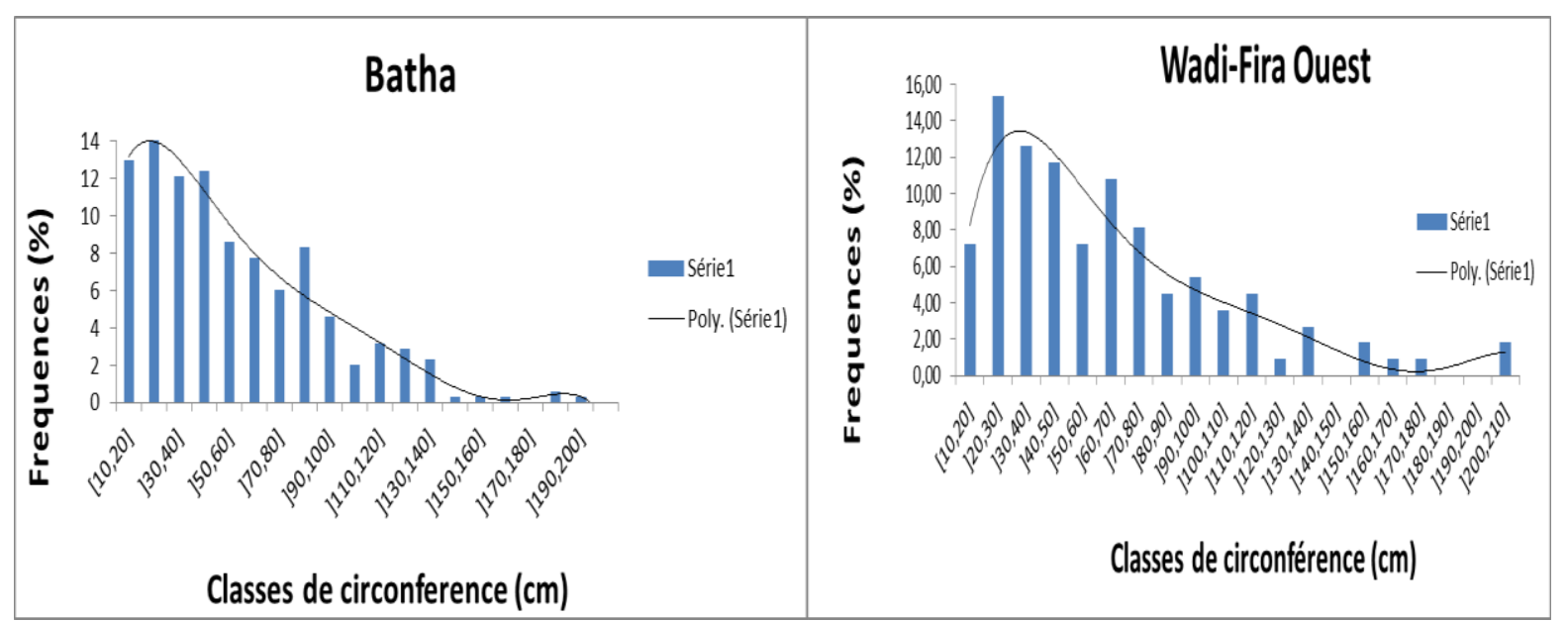

Figure 3 : Fréquence des individus selon les classes de circonférence (A) à Batha et (B) à Wadi-Fira Ouest. 


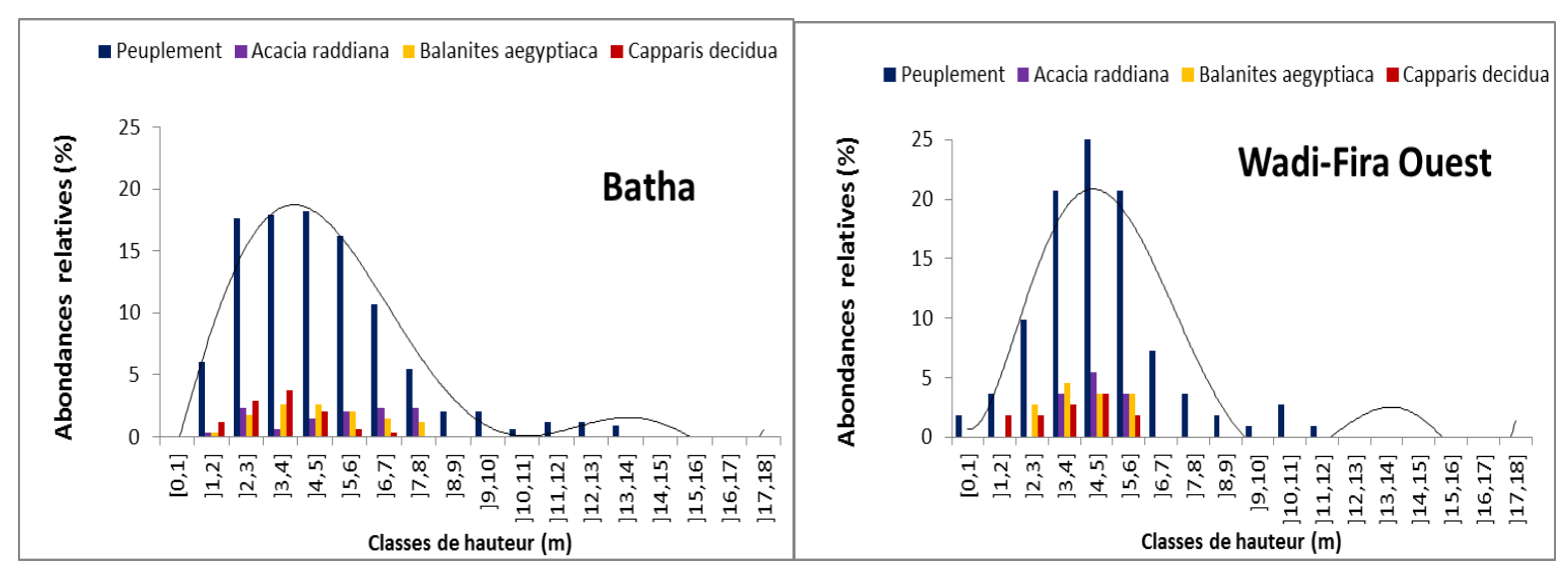

Figure 4 : Distribution des individus du peuplement et des espèces dominantes selon la hauteur (A) à Batha et (B) à Wadi-Fira Ouest.

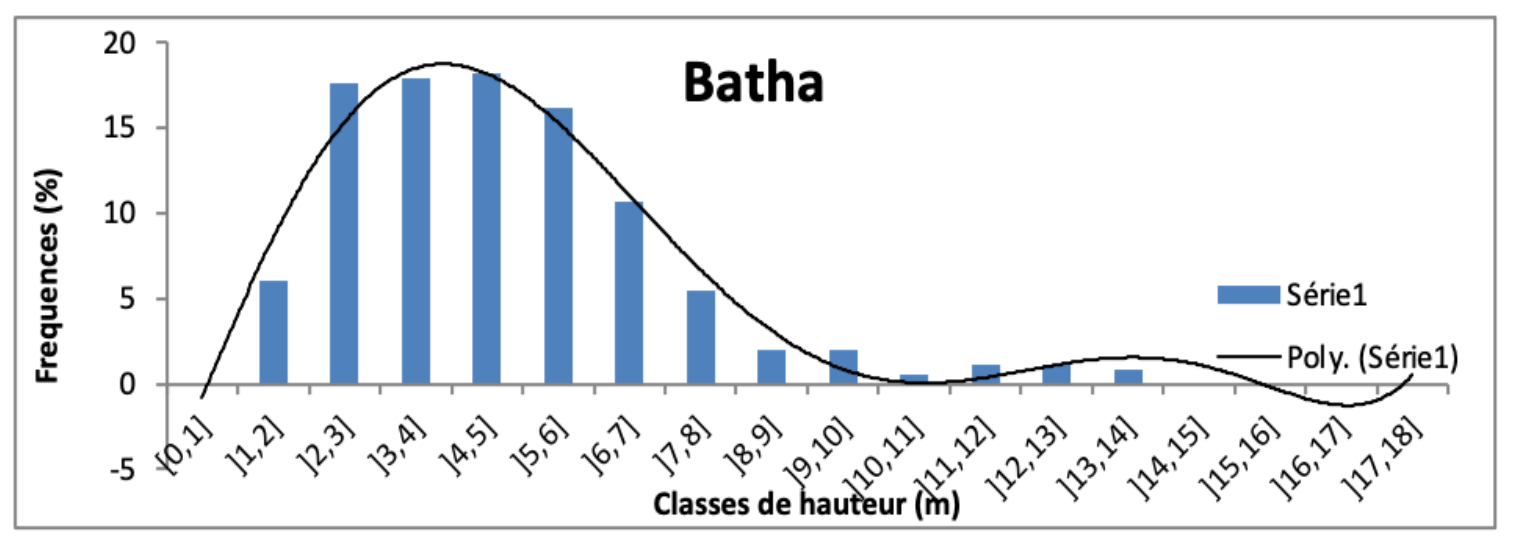

Figure 5 : Fréquence des espèces selon les classes de hauteur au Batha.

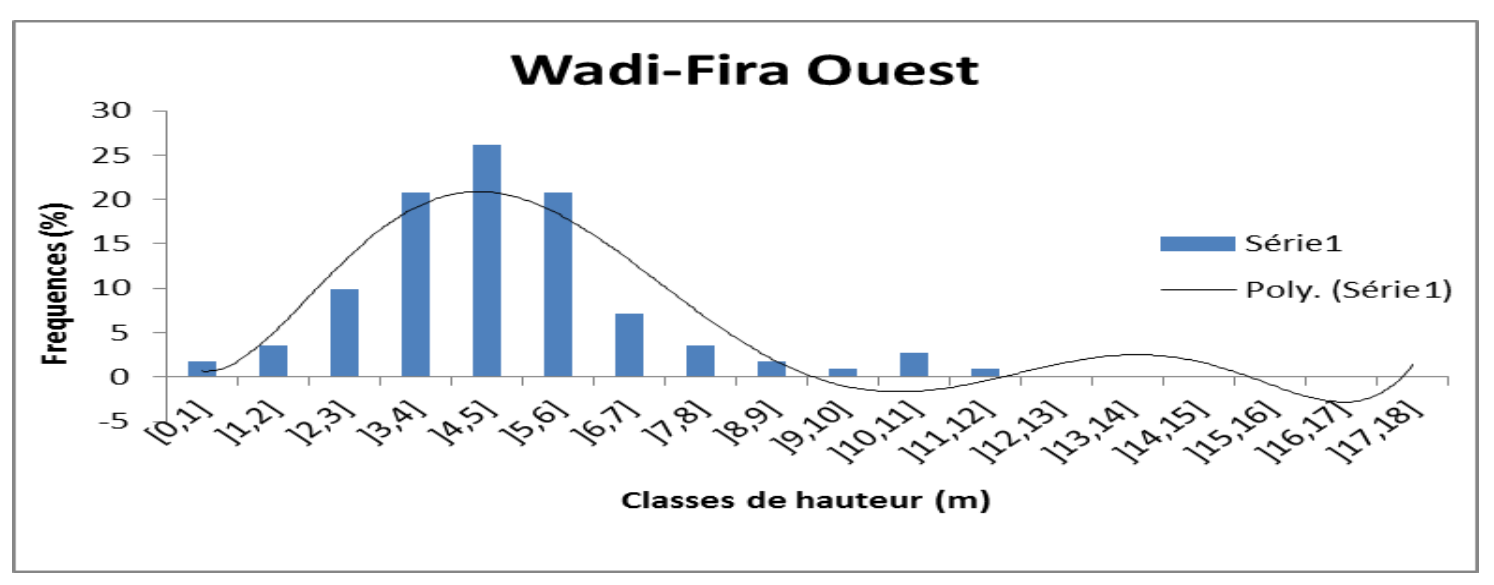

Figure 6 : Fréquence des espèces selon les classes de hauteur au Wadi-Fira Ouest. 


\section{DISCUSSION}

La présente étude a pour objectif de contribuer à la caractérisation des peuplements ligneux dans les sites de Batha et Wadi-Fira Ouest sur le tracé de la GMV au Tchad. Cette étude montre que la flore ligneuse inventoriée est riche de 15 espèces réparties dans 11 genres appartenant à 6 familles. De manière générale, la zone d'étude est pauvre en espèces comparée à d'autres études réalisées dans la zone sahélienne (Mahamat Saleh et al., 2015 a et b). La faible diversité traduit la dégradation des ressources dans ces zones (Niang et al., 2014).

La richesse floristique des sites étudiés est légèrement inférieure à celle obtenue par les travaux effectués au Tchad qui comptent 18 espèces, 15 genres et 9 familles (Mahamat Saleh et al., 2015b). Elle est légèrement élevée par rapport à celle du Guera au Tchad avec 11 espèces, 7 familles (Ngarnougber et al., 2017), des zones sahéliennes du Niger avec 10 espèces, 7 familles et 8 genres (Idrissa et al., 2020) et du Sénégal avec 12 espèces, 10 genres et 7 familles (Diallo et al., 2011). Cependant, elle est pratiquement identique à celle rencontrée au Niger qui présente 15 espèces, 11 genres et 10 familles (Ousseina et al., 2015). Cette différence serait due aux conditions climatiques extrêmes que connait le milieu ces dernières années d'une part et d'autre part à la pression anthropique et animale. L'effet combiné de ces facteurs fait régresser voire disparaitre de nombreuses espèces ligneuses (Idrissa et al., 2020).

Nous notons une dominance de la famille de Mimosaceae (6 espèces) et des Capparaceae (3 espèces). Cette dominance est due à la résistance des individus de ces familles aux stress climatiques d'une part (Bakhoum, 2013) et au fait que les espèces appartenant à ces familles sont du domaine saharo-sahélien d'autre part (Ousseina et al., 2015).

Les espèces les plus fréquentes dans la zone d'étude sont Balanites aegyptiaca (86,98\%), Acacia raddiana $(64,57 \%)$ et Capparis decidua (56,87\%). Les forts taux présentés par les espèces dominantes de la zone sont dus à l'adaptation de ces espèces aux conditions écologiques du milieu. Le peuplement ligneux ainsi que les espèces dominantes de la zone d'étude sont constitués des plus de $50 \%$ d'individus ayant moins de $60 \mathrm{~cm}$ de circonférence.

Les gros arbres sont minoritaires et les individus de petits diamètres sont majoritaires. Nos résultats s'accordent avec ceux de Ngaryo et al. (2010) et Mahamat Saleh et al. (2015b) qui ont travaillé dans la même zone, mais également avec ceux de Mohamed et al. (2008) dans la zone sahélienne. Cela se traduirait par une bonne capacité d'adaptation des espèces aux pressions du milieu notamment les feux de brousse et les fluctuations de saisons de pluie de ces dernières décennies (Goba et al., 2019) d'une part et la difficulté des arbres à croître normalement en épaisseur suite aux déficits hydriques cumulés (Diallo et al., 2011) d'autre part. Dans les sites, la distribution de Weibull à 3 paramètres du peuplement ligneux en fonction des classes de circonférence indique une distribution en «J» renversé, caractérisée par un nombre élevé d'individus juvéniles. Cette structure est dominée par des individus de faible diamètre qui représentent $51,08 \%$ des effectifs. Elle est caractéristique des formations arbustives sahéliennes et est considérée comme un indice de dégradation (Diallo et al., 2012). L'abondance des jeunes témoigne que les premières classes sont bien représentées (Idrissa et al., 2020).

Le peuplement du Batha est constitué de plus de $70 \%$ d'individus ayant une hauteur comprise entre 2 et $7 \mathrm{~m}$. Les espèces dominantes (Balanites aegyptiaca, Acacia raddiana et Capparis decidua) de ce site présentent des individus ne dépassant pas $8 \mathrm{~m}$ de hauteur. Tans dis que dans le Wadi-Fira Ouest, le peuplement est constitué de plus de $60 \%$ d'individus ayant entre 3 et $6 \mathrm{~m}$ de hauteur. Quant aux espèces dominantes, elles ont toutes une hauteur inférieure à $6 \mathrm{~m}$. Ces hauteurs caractérisent la végétation sahélienne, essentiellement arbustive (Diallo et al., 2012). Les individus de petites et 
grandes tailles sont peu fréquents et nos résultats confirment ceux de Djibo et al. (2018) dans le sahel.

La structure démographique des espèces en classe de hauteur révèle une prédominance des individus de petites et moyennes tailles ( 0,9 à $14 \mathrm{~m})$. La majorité des espèces se rencontrent dans les petites classes $[2,3 \mathrm{~m}] ;[3,4 \mathrm{~m}] ;[4,5 \mathrm{~m}]$ et $[5,6 \mathrm{~m}]$ avec des proportions respectives de $15,75 \%, 18,6 \%$, $20,13 \%$ et $17,29 \%$ de la population totale des individus recensés. Nos résultats s'accordent avec ceux de Ngaryo et al. (2010), Mahamat Saleh et al. (2015b) et de Ngarnougber et al. (2017) dans la même zone et sont identiques à ceux de Diallo et al. (2011) dans la zone sahélienne du Sénégal. L'abondance des individus de petites tailles serait due à la surexploitation qui entraine des stress et des traumatismes chez les individus (Kebenzikato et al., 2014).

\section{Conclusion}

L'étude de la distribution des ligneux selon la circonférence et les hauteurs dans le Batha et le Wadi-Fira Ouest, sur le tracé de la Grande Muraille Verte au Tchad a permis de montrer que la flore ligneuse inventoriée est riche de 15 espèces, réparties dans 11 genres et 6 familles. Ces travaux montrent que les structures en grosseur et en hauteur sont caractérisées par un grand nombre des petits arbres qui se rencontrent dans les basses classes. Les gros et les grands arbres sont peu fréquents. La strate ligneuse est dominée par des arbustes en grande partie. Les facteurs d'ordre climatique et anthropique ont un impact sur l'environnement de la zone d'étude. Les données obtenues de ces études ont permis de manière spécifique à déterminer la dynamique de peuplement des végétaux d'une part, et d'autre part, les relations entre ces derniers et le milieu physique. Il serait intéressant de poursuivre les investigations en étudiant la strate herbacée afin d'avoir une connaissance globale sur la flore de la zone d'étude mais également de concevoir une application pour suivre l'évolution du milieu.

\section{CONFLITS D'INTERETS}

Les auteurs déclarent qu'ils n'ont aucun conflit d'intérêts par rapport à cet article.

\section{CONTRIBUTIONS DES AUTEURS}

MAG est l'auteur principal de l'article ; il a assuré la conduite de travaux sur le terrain et la rédaction de la première version du manuscrit. MDD, AD et MMS ont contribué à la rédaction et à l'amélioration du manuscrit. AG a encadré et supervisé le travail.

\section{REMERCIEMENTS}

Les auteurs expriment leur profonde gratitude à l'Organisation Panafricaine d'Entraide Citoyenne (OPEC) qui a financé une partie de cette étude et les responsables du Secteur Sud des Aires Protégées de la Réserve de Faune de Ouadi Rimé-Ouadi Achim (Tchad).

\section{REFERENCES}

ANAM. 2020. Agence Nationale pour la Météorologie au Tchad. Paramètres météorologiques relatifs à la période 1987-2017 Ndjamena-Tchad Fiche de relevés climatiques $15 \mathrm{p}$.

Bakhoum A. 2013. Dynamique des ressources fourragères : indicateur de résilience des parcours communautaires de Tessekeré au Ferlo (Nord-Sénégal). Thèse de Doctorat unique en Biologie, Production et Pathologies animales, Option Ecologie Pastorale, FST-UCAD, $115 \mathrm{p}$.

Bechir AB, Mopate LY. 2015. Analyse de la dynamique des pâturages autour des ouvrages hydrauliques des zones pastorales du Batha Ouest du Tchad. Afrique SCIENCE, 11(1) : 212-226.

Berhaut J. 1979. Flore Illustrée du Sénégal (Tome VI). Gouvernement du Sénégal : Dakar ; 636 p.

Bernabe BP. 2013. Semi-quantitative evaluation of access and caverage. Région de Batha, Tchad. $55 \mathrm{p}$.

Boudet G. 1984. Manuel sur les Pâturages Tropicaux et les Cultures Fourragères 
(4e édn). Ministère de la Coopération, Manuels et Précis d'Elevage : Paris, 4: $1-25$.

Diallo A, Faye MN, Guisse A. 2011 a. Variations de la composition de la végétation herbacée des plantations de Acacia senegal (L.) WILLD de la zone de Dahra (Ferlo). Int. J. Biol. Chem. Sci., 5(3) : $\quad 1250-1264 . \quad$ DOI: 10.4314/ijbcs.v5i3.72273.

Diallo A, Faye MN, Guisse A. 2011 b. Structure des peuplements ligneux dans les plantations d'Acacia senegal (L.) Willd dans la zone de Dahra (Ferlo, Sénégal). Revue d'Ecologie : 14 p.

Diallo A, Agbangba EC, Thiaw A, Guisse A. 2012. Structure des populations de Acacia Senegal (L) Willd dans la zone de Tessékéré (Ferlo nord), Sénégal. Journal of Applied Biosciences, 59: 4366-4374.

Diallo MD, Goalbaye T, Mahamat-Saleh M, Agbangba EC, Ndao P, Doelsch E, Diop A, Guisse A. 2019. Influence des houppiers de quelques espèces végétales de la Grande Muraille Verte sur la biomasse microbienne et le taux d'azote minéral d'un sol ferrugineux tropical dans le Nord Ferlo, Sénégal. In La Grande Muraille Verte : une Réponse Africaine au Changement Climatique, Boetsch G, Duboz P, Guissé A, Sarr P (Eds). CNRS Editions : Paris ; 75-90.

Djibo I, Mani M, Chaibou I, Sarr O, Bakhoum A, Marichatou H, Akpo LE, Assane M. 2018. Caractéristiques de la végétation du parcours Gadoudhé, dans la commune rurale de Fabidji (Niger). International Journal of Biological and Chemical Sciences, 12(3): 1151-1163. DOI: https://dx.doi.org/10.4314/ijbcs.v12i3.7

Goba AE, Koff KG, Sié RS, Kouonon LC, Koff YA. 2019. Structure démographique et régénération naturelle des peuplements naturels de Pterocarpus erinaceus Poir. (Fabaceae) des savanes de Côte d'Ivoire. Bois et Forêts des Tropiques, 341 : 5-14. DOI: https://doi.org/10.19182/

bft2019.341.a31750

Goffner D, Jean-Luc P. 2020. La Grande Muraille Verte : un espoir pour reverdir le Sahel ? Encyclopédie de l'Environnement, url : https://www.encyclopedieenvironnement.org/vivant/grandemuraille-verte-afrique-espoir-reverdirsahel/.

Idrissa I, Morou B, Abdourhamane H, Karim S, Abdourhamane T, Djibo I, Mahamane A. 2020. Diversité floristique et structure démographique des peuplements ligneux des parcours naturels sahéliens du Sud-Est du Niger : Cas de l'enclave pastorale «Dadaria» (Mainé-Soroa, Diffa). Int. J. Biol. Chem. Sci., 14(3): 706-721. DOI : 10.4314/ijbcs.v14i3.6

INSEED. 2013. Deuxième enquête sur la consommation et secteur informel au Tchad-ECOSIT 3_Tchad, profil de pauvreté. Rapport final

Kaou Kak, Manzo OL, Dan Guimbo I, Karim S, Habou R, Paul R. 2017. Diversité floristique et structure de la végétation dans la zone dunaire du sud-est du Niger: cas de Mainé Soroa. Journal of Applied Biosciences, 120(1): 14 p. DOI: 10.4314/jab.v120i1.8

Kebenzikato AB, Wala K, Dourma M, Atakpama W, Dimobe K, Pereki H. 2014. Distribution et structure des parcs à Adansonia digitata L. (baobab) au Togo. Afrique Science, 10(2) : 434 - 449.

Lebrun JP, Stork A. 1997. Enumération des Plantes à Fleurs d'Afrique Tropicale (Vol IV). Edition des Conservatoires et Jardin Botaniques de la Ville de Genève : Genève ; 711 p.

Maydell HJV. 1983. Arbres et Arbustes du Sahel. Office Allemand de la Coopération Technique, GTZ: Eschborn, Allemagne ; 531p.

Mahamat-Saleh M, Diallo MD, Niang K, Sagna MB, Ndiaye O, Guisse A. 2015 a. Tracé de la Grande Muraille Verte du Tchad : relation de régression entre la 
hauteur et la circonférence des arbres. Afrique Science, 11(6) : 14 - 20.

Mahamat-Saleh M, Ndiaye O, Diallo MD, Goy S, Niang K, Diallo A, Guisse A. 2015 b. Caractérisation des peuplements ligneux sur le tracé de la Grande Muraille Verte au Tchad. Int. J. Biol. Chem. Sci., 9(5): 2617-2627. DOI : 10.4314/ijbcs.v9i5.31

Mahamat-Saleh M, Diallo MD, Sarr PS, Ndiaye O, Goalbaye T, Niang K, Goy S, Ndiaye D, Guisse A. 2016. Diversité floristique des peuplements ligneux des trois sites du tracé de la grande muraille verte du Tchad. Annales des Sciences Agronomiques, 20(2): 1-12.

Mohamed MC, Lo M, Bassene E, Akpo LE. 2008. Caractéristiques de la flore et de la végétation ligneuses de forêts communautaires de la zone soudano sahélienne au Sénégal. Journal des Sciences et Technologies, 6(2) : $72-85$.

Ndiaye O, Diallo A, Sagna MB, Guisse A. 2013. Diversité floristique des peuplements ligneux du Ferlo, Sénégal. Vertigo, la revue électronique en sciences de l'environnement, 13(3) : 12p.

Ndong AT, Ndiaye O, Sagna MB, Diallo A, Galop D, Guisse A. 2015. Caractérisation de la végétation ligneuse sahélienne du Sénégal: cas du Ferlo. International Journal of Biological and Chemical Sciences, 9(6): 2582-2594. DOI:

http://dx.doi.org/10.4314/ijbcs.v9i6.6

Ngarnougber C, Ngaryo FT, Adamou I. 2017. Caractérisation des ligneux de la savane sahélienne à Acacia senegal (L) Willd dans la région du Guéra, Tchad. International Journal of Applied Research, 3(4): 600-606.

Ngaryo FT, Goudiaby VC, Akpo LE. 2010. Caractéristiques d'une gommeraie d'Acacia senegal (L.) Wild. dans la région du Chari Baguirmi au Tchad. Journal des Sciences, 10 (2) : 13 - 23.

Ngom D, Boubacar C, Sagna B, Gomis ZD. 2018. Cortège floristique, paramètres structuraux et indicateurs d'anthropisation des parcs agroforestiers à Elaesis guineensis Jacq. en basse Casamance, Sénégal. Journal of Animal and Plant Sciences, 36(3) : 5919-5932.

Niang K, Ndiaye O, Diallo A, Guisse A. 2014. Flore et structure de la végétation ligneuse le long de la Grande Muraille Verte au Ferlo, nord Sénégal. Journal of Applied Biosciences, 79: 6938 - 6946.

Ousseina S, Fortina R, Marichatou H, Yenikoye A. 2015. Diversité, structure et régénération de la végétation ligneuse de la Station Sahélienne Expérimentale de Toukounous, Niger. International Journal of Biological and Chemical Sciences, 9(2): 910-926. DOI: http://dx.doi.org/10.4314/ijbcs.v9i2.29

Sarr O, Diatta AS, Gueye M, Ndiaye PM, Guisse A, Akpo LE. 2013. Importance des ligneux fourragers dans un système agropastoral au Sénégal (Afrique de l'Ouest). Revue Méd. Vét., 164 (1) : 2-8.

Sinsin B, Kampmann D. 2010. Atlas de la biodiversité de l'Afrique de l'Ouest, Tome I: Benin. Cotonou et Frankfurt/Main. BIOTA, 676 P.

SNPA/GMV. 2012. Stratégie nationale et plan d'actions pour la mise en œuvre de l'initiative grande muraille verte au Tchad.70 p.

Talla R, Sagna MB, Diallo MD, Diallo A, Faye N, Sarr S, Badji ES, Diatta S, Ngom D, Guisse A. 2020. Population Structure and Toposequence Distribution of Boscia senegalensis (Pers.) Lam. ex Poir and Sclerocarya birrea (A. Rich) Hoscht in the Ferlo (Senegal). Journal of Plant Sciences, 8(5): 167-176.

Touffet J. 1982. Dictionnaire Essentiel d'Ecologie. Edité par Ouest France ; $108 \mathrm{p}$. 